BIPOLAR FLOWS, JETS, AND PROTOSTARS 


\title{
STAR FORMATION AND THE CIRCUMSTELLAR MATTER OF YOUNG STELLAR OBJECTS
}

\author{
Frank H. Shu and Fred C. Adams
}

Astronomy Department, University of California, Berkeley CA 94720, USA

\begin{abstract}
We propose that the formation of low mass stars in molecular clouds takes place in four stages. The first stage is the formation of slowly rotating cloud cores through the slow leakage of magnetic (and turbulent) support by ambipolar diffusion. These cores asymptotically approach quasistatic states resembling singular isothermal spheres, but such end states cannot actually be reached because they are unstable. The second phase begins when a condensing cloud core passes the brink of instability and collapses dynamically from "insideout," building up a central protostar and nebular disk. The emergent spectral energy distributions of theoretical models in the infall stage are in close agreement with those of recently found infrared sources with steep spectra. As the rotating protostar gains mass, deuterium will eventually ignite in the central regions and drive the star nearly completely convective if its mass is less than about $2 M_{\odot}$. This initiates the next step of evolution - the bipolar outflow phase - in which a stellar wind pushes outward and breaks through the infalling envelope. The initial breakout is likely to occur along the rotational poles, leading to collimated jets and bipolar outflows. The intense stellar wind eventually widens to sweep out gas in nearly all $4 \pi$ steradian, revealing the fourth stage - a T Tauri star with a surrounding remnant nebular disk. Radiation from a disk adds an infrared excess to the expected spectral energy distribution of the revealed source. The detailed shape of this infrared excess depends on whether the disk is largely passive and merely reprocesses stellar photons, or is relatively massive and actively accreting. Both extremes of spectral shapes are observed in T Tauri stars; the amount of circumstellar material in the form of disks around nearly formed stars may be related to the dual issues of the origins of binary-star and planetary systems.
\end{abstract}

\section{INTRODUCTION}

Star formation occurs today primarily in large molecular clouds which have masses of $10^{4}-10^{6} M_{\odot}$ (Zuckerman and Palmer 1974, Burton 1976, Solomon and Sanders 1985). In contrast, the masses of the stars which result from the process are commonly a solar mass or less. This raises one of the basic questions of star formation - Why does the interstellar medium, which has ample raw material to produce very massive objects, mostly produce self-gravitating balls of gas that are only marginally capable of thermonuclear fusion? 
This question is highlighted by the realization that star formation in molecular clouds is generally an inefficient mechanism; usually, only $\sim 1 \%$ of the bulk matter in a molecular cloud is transformed into stars during a generation of star birth (see, e.g., Scoville 1986). This inefficiency results in a lot of material being left over in the immediate vicinity of the formed star; associated with young stellar objects (YSOs) is a wide variety of forms of circumstellar matter: infraredemitting shells, disks, jets, Herbig-Haro objects, bipolar (molecular) lobes, and cometary (reflection) nebulae. What determines whether a given parcel of matter becomes incorporated into the newly formed star, or is left over as debris from the star formation process? What accounts for the diversity of forms of circumstellar matter?

This paper approaches the above questions by considering a comprehensive picture of star formation. The theory schematically breaks down the complete process into four evolutionary phases. The first phase is the formation of molecular cloud cores - centrally condensed regions within larger molecular clouds. Since the existence of such entities is fairly well established observationally (see Myers and Benson 1983), and since the theory of core formation by ambipolar diffusion has been discussed elsewhere (see, e.g., Lizano and Shu 1986), we limit our discussion to the three later stages of evolution. We shall also confine our attention to the kinds of cloud cores which lead to the birth of low mass stars, the extreme conditions needed to produce high mass stars being more controversial (cf. Appenzeller and Tscharnuter 1974; Klein, Whitaker, and Sandford 1985; Shu 1986).

The second phase of star formation begins when a molecular cloud core becomes unstable and starts to collapse. This evolutionary phase is characterized by the existence of a central protostar and disk with a surrounding infalling envelope of dust and gas. We discuss the hydrodynamical properties of such objects in $\S I I$, their evolution in §III, and the corresponding emergent spectra in §IV.

As a low mass protostar accretes matter, it naturally evolves toward a state with a stellar wind, but at first the ram pressure from material falling directly onto the stellar surface suppresses breakout. Gradually, the "lid" of direct infall will weaken as the incoming material falls preferentially into the disk rather than onto the star. The stellar wind then rushes through the channels of weakest resistance (the rotational poles), and the protostar enters the bipolar flow phase. We discuss this third evolutionary phase in $§ \mathrm{~V}$.

Eventually, the spreading stellar wind will reverse the infall over almost the entire celestial sphere centered on the object. The object then enters the final phase of protostellar evolution and becomes a newly revealed T Tauri star with a surrounding nebular disk. The theory of the infrared excesses associated with such configurations is outlined in §VI.

There is a fifth phase which is not addressed by us, namely, the final disappearance of the nebular disk matter as it is incorporated into the bodies of planetary or stellar companions, or is dispersed and eroded by the energetic outflow. The topic of "naked T Tauri stars" (Mundt et al. 1983, Walter 1986) more properly belongs to the subject of pre-main-sequence evolution than to star formation, although it 
can also be viewed as logically flowing from the developments of the four prior stages.

\section{INSIDE-OUT COLLAPSE OF UNSTABLE CLOUD CORES}

As a starting point for the present discussion, we assume that the density distribution $\rho$ of a molecular cloud core which has reached the brink of gravitational instability can be approximated as a singular isothermal sphere, whose self-gravity is exactly but precariously balanced by a thermal pressure gradient (Chandrasekhar 1939):

$$
\rho=\frac{a^{2}}{2 \pi G r^{2}}
$$

where $a=(k T / m)^{1 / 2}$ is the isothermal sound speed and $T$ and $m$ are the (constant) temperature and mean molecular weight of the gas. (If magnetic fields and turbulence make non-negligible contributions to the mechanical support before collapse, we assume their effects can be mimicked by appropriately redefining an "effective" sound speed a.) Bodenheimer and Sweigart (1968) showed that subsonic evolution of a non-rotating, non-magnetic cloud would always tend to produce a $\rho \propto r^{-2}$ density distribution (see also Larson 1969). Shu (1977) discovered that the singular isothermal sphere (1), long known to be unstable to gravitational contraction, has a self-similar collapse solution, so that the solution at any instant in time looks like the solution at a previous instant except for scaling factors. The form of the similarity solution could be found analytically apart from the integration of some simple ordinary differential equations. Since the central density of the singular isothermal sphere is infinite, the adoption of equation (1) for the initial configuration of the collapse problem has been criticized as being artificial by Whitworth and Summers (1985). However, Nakano (1981) found that including grain coupling in ambipolar diffusion calculations (Elmegreen 1979, 1986; Nakano and Umebayshi 1980), produces very high densities (compared to envelope values) in the central regions of a molecular cloud core before dynamical collapse occurs. As long as the power-law portion of a real core solution spans several decades in density, its gravitational collapse is likely to be well represented by the self-similar solution for the singular isothermal sphere.

Notice that equation (1) asserts that there is no such thing as a typical density for a molecular cloud core - a power law has no characteristic scale. In particular, equation (1) predicts that the sizes of the maps obtained by radio-line observers will depend on the molecular transition studied; lower density tracers should produce larger maps than higher density tracers, in rough qualitative and quantitative agreement with the actual observations (Myers 1987, Walmsley 1987). Because equation (1) has no characteristic density scale, it has no characteristic Jeans mass - in fact, every radius $r$ contains about one Jeans mass, $2 a^{2} r / G \propto a^{3} \rho^{-1 / 2}$. In Taurus, where $a \approx 0.2 \mathrm{~km} / \mathrm{s}, 1 M_{\odot}$ is contained roughly within $r=0.05 \mathrm{pc}$ where $n_{\mathrm{H}_{2}} \sim 10^{4} \mathrm{~cm}^{-3}$ (cf. Myers and Benson 1983); in Ophiuchus, where $a \approx 0.35$ $\mathrm{km} / \mathrm{s}, 1 M_{\odot}$ is contained roughly within $r=0.02 \mathrm{pc}$ where $n_{\mathrm{H}_{2}} \sim 10^{5}-10^{6} \mathrm{~cm}^{-3}$ 
(cf. Martin-Pintado et al. 1983; Zeng, Batrla, and Wilson 1984; Wadiak et al. 1985). Going to lower density contours than traced by $\mathrm{NH}_{3}$ or $\mathrm{H}_{2} \mathrm{CO}$ or $\mathrm{HCO}^{+}$, e.g., as defined by various isotopes of the $\mathrm{CO}$ molecule, gives larger maps and larger masses.

In any case, with the adoption of equation (1) to characterize the core before it undergoes gravitational collapse, there is no unique mass scale that can be associated with the resulting star. Instead of a mass, it is the rate at which the central object accumulates matter through infall which is well defined; in the selfsimilar solution, this rate is given by

$$
\dot{M}=m_{0} a^{3} / G
$$

where $m_{0}=0.975$. For $a=0.2 \mathrm{~km} / \mathrm{s}, \dot{M}=2 \times 10^{-6} M_{\odot} / \mathrm{y} ;$ for $a=0.35 \mathrm{~km} / \mathrm{s}$; $\dot{M}=1 \times 10^{-5} M_{\odot} / y$.

In the self-similar collapse solution, infall is initiated from "inside-out" by an expansion wave which propagates outward at the speed of sound into the ambient (static) molecular cloud core. The head of the expansion wave reaches the radius $r_{h}=a t$ in time $t$; and supersonic inflow velocities are generated interior to $r_{0} \approx$ 0.4 at, inside of which the density begins to approach the free-fall form,

$$
\rho=C r^{-3 / 2}
$$

where $C \equiv \dot{M} / 4 \pi(2 G M)^{1 / 2}$.

The above discussion applies only to purely spherical collapse. When rotation is included in the problem as a small perturbational effect, the axisymmetric timedependent collapse (with variations in two spatial dimensions) can still be followed analytically (Terebey, Shu, and Cassen 1984). In the inner parts the rotationallymodified similarity solution asymptotically joins onto free-fall ballistic trajectories, which are parabolae in the limit of a very concentrated mass distribution for the central object (star plus disk). The density distribution (3) is a good approximation outside a centrifugal radius,

$$
R_{C} \equiv G^{3} M^{3} \Omega^{2} / 16 a^{8},
$$

the position where infalling matter in the equatorial plane encounters a centrifugal barrier if it conserves its initial specific angular momentum. In equation (4), $M=$ $\dot{M} t$ is the infallen mass, and $\Omega$ is the assumed (constant) rotation rate of the cloud core before collapse. Inside $R_{C}$ the isodensity contours become highly flattened and increase less steeply inward as a spherical average than in the nonrotating case. When averaged over all angles, the density increases inward of $\boldsymbol{R}_{C}$ approximately as $\rho \propto r^{-1 / 2}$. When $R_{C}$ is greater than the stellar radius $R_{*}$, a nebular disk forms around the protostar whose outer dimension extends at least to $R_{C}$ (beyond, if the disk has internal mechanisms for appreciable transport of mass and angular momentum in an infall time scale $M / \dot{M})$. 


\section{PRotostellar evolution}

When the infalling dust and gas become optically thick to the emergent infrared radiation, the isothermal approximation begins to break down badly. In models of low-mass protostars where the effects of rotation are ignored, this occurs inside a radius $r_{e}$ of approximately $10^{14} \mathrm{~cm}$ (Stahler, Shu, and Taam 1980a,b; 1981; hereafter SST). Fortunately, within the region bounded by the the dust photosphere, $r_{e}$, and the stellar radius, $R_{*} \sim 10^{11.5} \mathrm{~cm}$ (defined by the accretion shock), the material falls nearly freely toward the mass at the center. Hence, the crossing time for both matter and radiation is short compared to the evolutionary time, and a condition of steady-state flow holds to a high degree of approximation. The inner region of complicated radiative hydrodynamics may thus be solved (using carefully chosen closure relations for the frequency-integrated moment equations of the radiation field) as a set of ordinary differential equations, greatly simplifying the computational demands of the problem. For this inner problem, the similarity solution discussed in the previous section provides outer boundary conditions, namely, inflow at free-fall speeds with mass infall rate (2).

Using an infall rate $\dot{M}=1 \times 10^{-5} M_{\odot} / \mathrm{y}$, SST found that the protostar accumulated matter (processed through an accretion shock) of ever increasing specific entropy. Thus, the star remained radiative until deuterium ignited near the center when the stellar mass was about $0.3 M_{\odot}$. A convection zone then spread outward through the star until it became almost entirely convective at a mass of about $0.5 M_{\odot}$. Except for this event, nothing happened to distinguish a particular mass scale for the accreting protostar; in the actual calculations, the infall was artificially terminated after $10^{5} \mathrm{y}$ when the star had accumulated $1 M_{\odot}$. The surface of the star, which had been kept abnormally hot and luminous by a standing shock, then cooled in less than a day and joined a convective pre-main-sequence track (Hayashi, Hoshi, and Sugimoto 1962). The disappearance of the infall region would first make the star optically visible at this point. If the loci of such points in the Hertzsprung-Russell diagram for different masses are joined, the result is a "birthline" for pre-main-sequence stars of low mass. Stahler (1983) showed the birthline corresponding to spherical mass accretion at the rate $\dot{M}=1 \times 10^{-5} M_{\odot} / \mathrm{y}$ gave a remarkable fit to the upper envelope for $T$ Tauri stars in Taurus-Auriga, Orion, NGC 7000/IC 5070, and Ophiuchus (Cohen and Kuhi 1979).

Unfortunately, the agreement between the theoretical birthline and the observed one can be subjected to a number of criticisms (cf. Mercer-Smith, Cameron, and Epstein 1984). First, the results described above are limited to purely spherical infall; even a small amount of rotation in the original molecular cloud core would have produced accretion partially through a disk. Second, although the adopted infall rate of $1 \times 10^{-5} M_{\odot} / y$ is appropriate for Ophiuchus, it is $\sim 5$ times too large for Taurus. Using a scaling law $R_{*} \propto \dot{M}^{1 / 3}$ appropriate to stars which derive all of their energy from accretion (see SST's comparison with Winkler and Newman 1980), the spherical-collapse birthline for Taurus becomes underluminous by a factor of $\sim 3$. 
Shu (1985, see also Shu and Terebey 1984) pointed out that the observed birthline for $\mathrm{T}$ Tauri stars can be understood, not as a special result of a spherically symmetric scenario for star formation, but in terms of an end condition that the star must be able to burn deuterium in order to clear the shroud of placental material. The basic idea is that when the convection driven by deuterium burning in a low mass protostar is coupled with the presence of differential rotation, dynamo action results (Parker 1979), which taps the rotational energy stored in the star. The released energy is speculated to power the intense stellar surface activity observed in young stellar objects. The resulting stellar wind eventually reverses the infall and allows the newly formed star to become visible.

The luminosity of such an object includes sources other than accretion. The primary consideration in this case is that for any given mass, there is a unique radius for which a completely convective star will have a central temperature (Chandrasekhar 1939),

$$
T_{\mathrm{c}}=0.54 G M_{*} \mu H / k R_{*},
$$

high enough for deuterium to burn. In equation (5) we have assumed that the convective star is an ideal gas of constant mean molecular weight $m \equiv \mu H$ (i.e., a polytrope of index 1.5). For cosmic abundances and $0.01 M_{\odot}<M_{*}<2 M_{\odot}$, we can obtain an approximate criterion for deuterium burning by setting $T_{\mathrm{c}}=1 \times 10^{6}$ $\mathrm{K}$ in equation (5):

$$
R_{*} / R_{\odot} \approx 0.15+7.6\left(M_{*} / M_{\odot}\right) \text {, }
$$

where the small correction term 0.15 on the right-hand side enters to take into account the effects of partial degeneracy at low stellar masses (cf. Nelson, Rappaport, and Joss 1986). Stahler (1986, private communication) points out that the derivation of equation (6) should really include the notion that deuterium burning occurs in steady state; however, the required modification is small because the reaction rate is extremely sensitive to temperatures near $1 \times 10^{6} \mathrm{~K}$.

Equation (6) yields a birthline in the Hertzsprung-Russell diagram for pre-mainsequence stars which does not differ much from that drawn originally by Stahler (1983). The main difference is that we have now identified the specific mechanism responsible for the unveiling of low-mass protostars and that this mechanism defines a birthline for pre-main-sequence evolution through physics (thermonuclear fusion and stellar structure) that is relatively insensitive to the detailed manner in which central mass is accumulated (i.e., by direct infall or disk accretion).

The rate of stellar mass accumulation $\dot{M}_{*}$ affects only the timing of deuterium ignition, i.e., the eventual mass of the star $M_{*}$ on the birthline. When $\Omega \neq 0$, the rate of stellar mass accumulation,

$$
\dot{M}_{*}=\dot{M}_{*}(\text { direct infall })+\dot{M}_{*}(\text { disk accretion })
$$

does not equal the total infall rate $\dot{M}$ given by equation (2) unless all of the mass which falls into the disk is shortly incorporated into the central star (i.e., unless the parameter $\eta_{D}$ to be discussed below equals unity). Higher rates of stellar 
mass accumulation allow less time for the radiation of the stellar binding energy, leading to larger values of $R_{*}$ at every $M_{*}$, and thus producing higher masses before the onset of deuterium burning. From computed radiation losses of premain-sequence stars on Hayashi contraction tracks, we estimate that an average rate of $\dot{M}_{*}=10^{-7} M_{\odot} / \mathrm{y}$ will produce a protostar which begins to drive a wind after it accumulates $\sim 0.1 M_{\odot} ; 10^{-6} M_{\odot} / y$, after $\sim 0.3 M_{\odot} ; 10^{-5} M_{\odot} / y$, after $\sim 0.5 M_{\odot}$; and $10^{-4} M_{\odot} / y$, after $\sim 2 M_{\odot}$. The final stellar masses could be higher than the above listed values if the infall is sufficiently intense to suppress a breakout of the stellar wind despite the convection induced by deuterium burning or if significant amounts of stellar accretion still occurs from a disk during the bipolar flow phase of protostellar evolution.

In any case, for stars which accumulate $\sim 2 M_{\odot}$ before deuterium ignites, the extra interior luminosity released by deuterium burning can be carried out by radiative diffusion without the inducement of convection. The termination of stellar accumulation for high mass stars must therefore occur by a different process than outlined above (see also the discussion of Cassen, Shu, and Terebey 1985). Since $\dot{M}_{*}$ cannot on average exceed the total infall rate $\dot{M}$, equation (2) implies that the production of high mass stars requires effective values of $a$ greater than or comparable to $0.7 \mathrm{~km} / \mathrm{s}$ if $\dot{M}$ is to exceed $10^{-4} M_{\odot} / \mathrm{y}$. This requires gas temperatures in excess of $100 \mathrm{~K}$ in the original molecular cloud core if the primary contribution to $a$ is thermal (but non-negligible magnetic and "turbulent" contributions are likely to be also present). A possible route for obtaining warm molecular cloud cores and high effective values of $a$ is discussed by Shu, Lizano, and Adams (198\%), by Shu (1986), and by Lizano and Shu (1986).

\section{INFRARED EMISSION FROM PROTOSTARS}

In order to test the fundamental notion that stars form through a process of infall, it is helpful to calculate the expected emergent spectral energy distributions of protostars. Spherically symmetric calculations have been performed by numerous groups (Larson 1969, Yorke and Krugel 1977, Bertout and Yorke 1978, Yorke and Shustov 1981), and Adams and Shu (1985, 1986; hereafter AS) have developed a fast approximate technique applicable to multi-dimensional situations as well.

The AS models are characterized by five parameters: the isothermal sound speed $a$ of the initial cloud core, its angular velocity $\Omega$, the mass $M=M_{*}+M_{D}$ that has accumulated to date in the central star and disk, and the efficiencies $\eta_{*}$ and $\eta_{D}$ with which the star and disk dissipate the energy of differential rotation. Given a deduced value of $a$, the quantities $M, \eta_{*}$, and $\eta_{D}$ combine to produce a single observable - the total protostellar luminosity $L$. In practice, we fixed $\eta_{*}$ and $\eta_{D}$ at standard values, and the mass $M$ was varied in order to match the observed luminosity $L$. This left a single free parameter, the cloud angular velocity $\Omega$, with which to fit the spectrum. The resulting theoretical spectral energy distributions gave good agreement with observations of a class of lowluminosity infrared sources, those which have steep spectra and are found near 

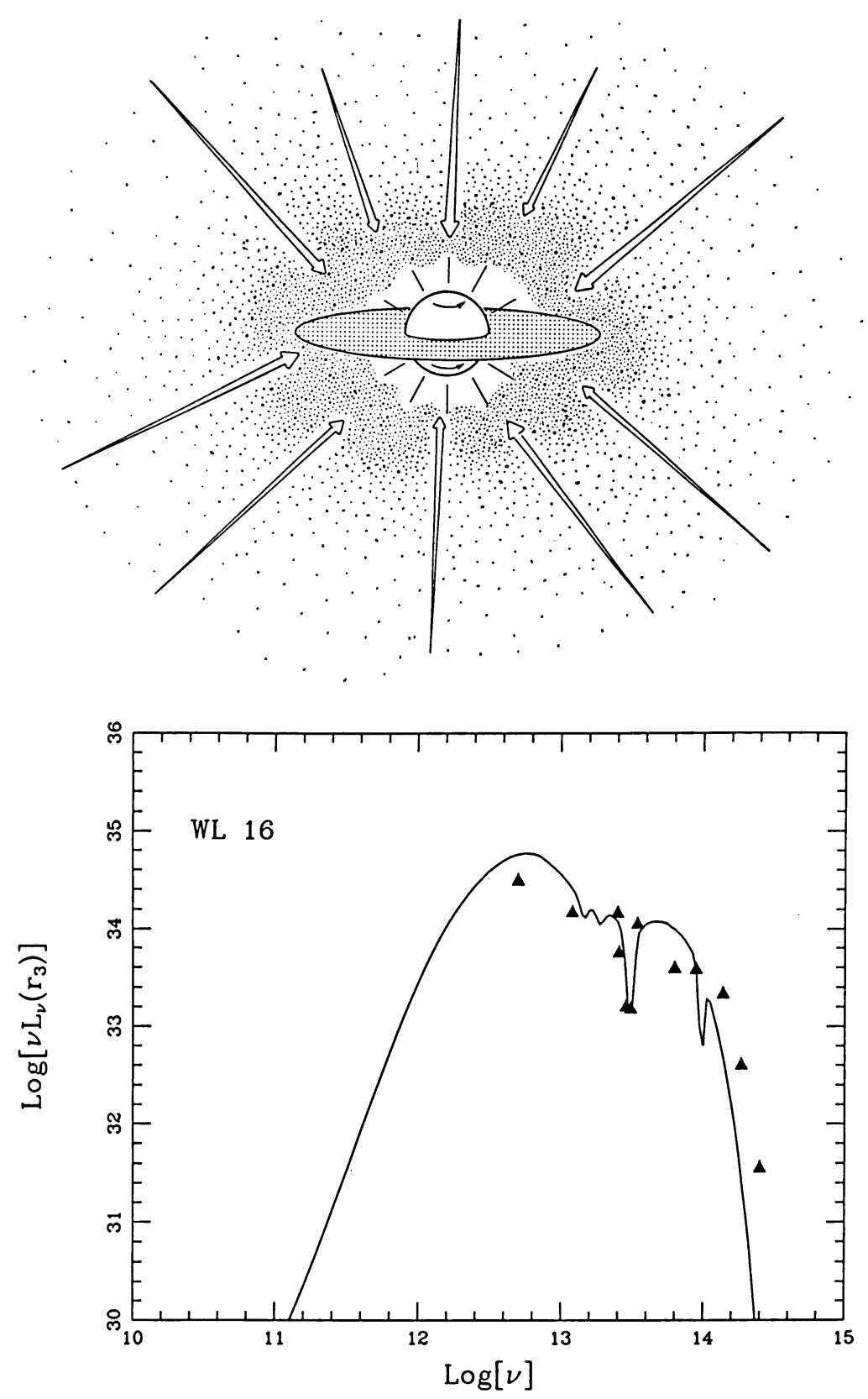

Figure 1. Comparison of theoretical and observed emergent spectral energy distributions of protostellar candidate WL 16 (Ophiuchus): data taken from Wilking and Lada (1983), Lada and Wilking (1984), and Young, Lada, and Wilking (1986); theoretical model assumes $a=0.35 \mathrm{~km} / \mathrm{s}, M=0.5 M_{\odot}$ and $\Omega=5 \times 10^{-13} \mathrm{rad} / \mathrm{s}$; (all quantities are given in cgs units). 
the centers of dense molecular cloud cores (see Figure 1 for an example). The angular velocities required in order to match the observed emission spectra of seven protostellar candidates (see Adams, Lada, and Shu 1987; hereafter ALS) lie in the range $\Omega=2 \times 10^{-14} \mathrm{~s}^{-1}$ to $5 \times 10^{-13} \mathrm{~s}^{-1}$. Such rates are compatible with measured values (e.g., Myers and Benson 1983, Wadiak et al. 1985, Goodman 1986), lending support to the entire theoretical development.

The shape of the emergent spectral energy distributions is especially sensitive to the centrifugal radius $R_{C}$ defined by equation (4). To a good approximation, the total extinction to the central source along a typical line of sight is given by

$$
A_{V} \approx 3.5 \kappa_{V} C R_{C}^{-1 / 2}
$$

where $\kappa_{V}$ is the opacity at visual wavelenths $\left(\approx 200-250 \mathrm{~cm}^{2} / \mathrm{g}\right)$. The column density of matter reprocessing the stellar and disk photons to radiation of longer wavelengths is the primary determinant of both the depth of the $10 \mu \mathrm{m}$ silicate feature and the overall spectral breadth (see ALS).

\section{BIPOLAR OUTFLOW PHASE}

The rotating infall models described above have the interesting property that they correctly predict the emergent spectra for some infrared sources which are known bipolar outflow sources, i.e., objects that have entered the third phase of protostellar evolution (see Figure 2 for an example). This finding supports the idea that well-collimated sources represent objects in which inflow and outflow are taking place simultaneously. Thus, these objects yield the transitional phase of evolution between a purely accreting protostar and a fully revealed pre-mainsequence star (Shu and Terebey 1984; Cassen, Shu, and Terebey 1985).

Observationally, of course, outflows from young stellar objects are known often to take a well collimated form. At least two manifestations of the phenomenon are known: long and narrow optical jets (e.g., Mundt and Fried 1983) and CO bipolar outflows (e.g., Bally and Lada 1983). A complete theory of star formation should explain the origins of both kinds of features, as well as establish if there is a relation between them.

The general notion that stellar outflows from young stars should be directed in two diametrically opposed directions finds a natural explanation in the scenario for star formation posed above. During the infall phase, the swirling inflowing matter acts like the lid of a pressure cooker and suppresses any incipient stellar outflow. As the lid weakens, breakout will occur through the channel of least resistance (the safety valve) at the rotational pole(s) of the accreting protostar where the total column of infalling material is least (see Figure 6 of Terebey, Shu, and Cassen 1984). Since the system is smoothly transforming from a subcritical state (inflow) to a supercritical state (outflow), breakout in the absence of perfect spherical symmetry should first occur at one point (on each hemisphere). Thus, 

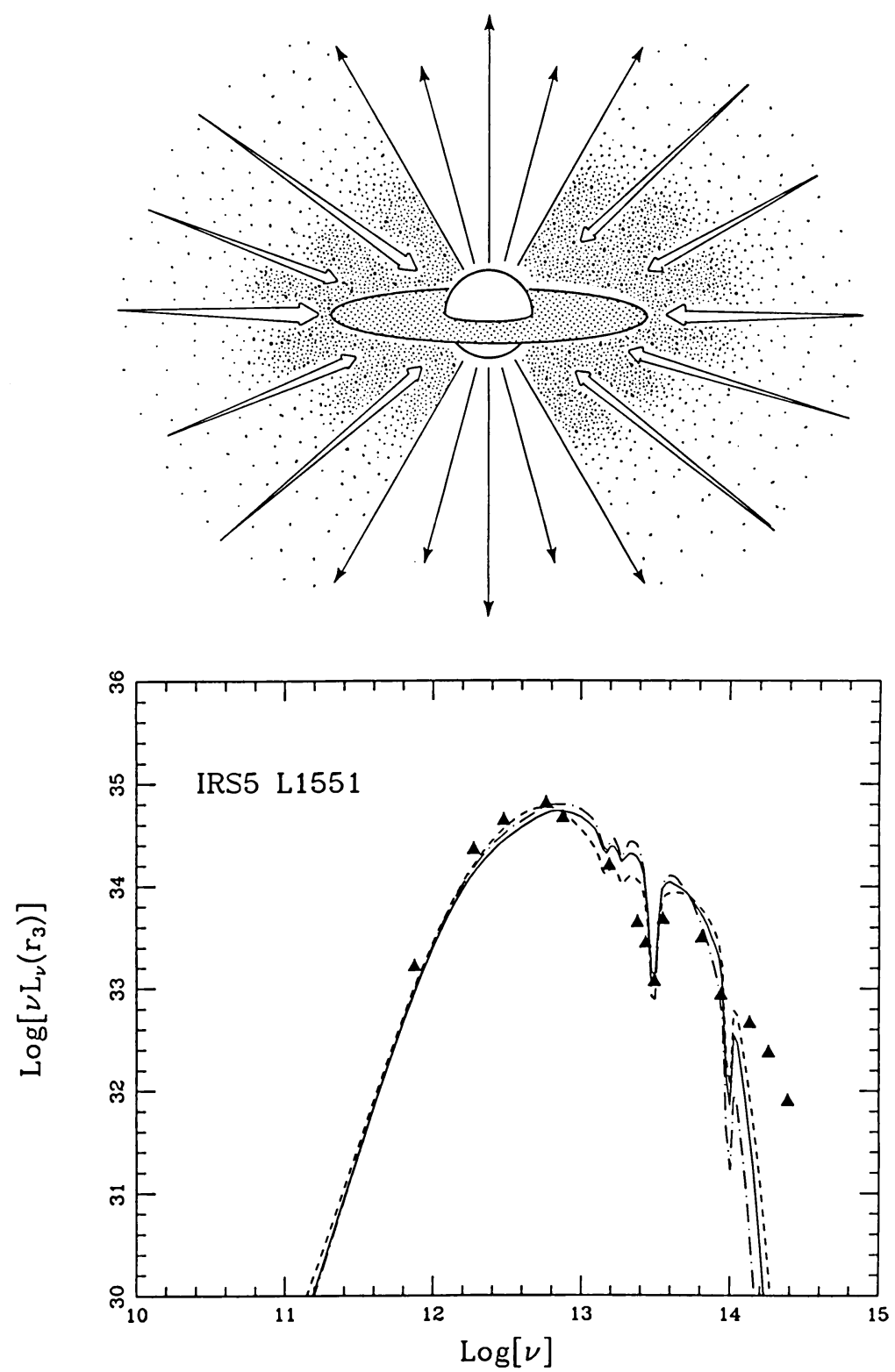

Figure 2. Theoretical and observed spectrum of bipolar outflow source IRS5 L1551 (Taurus): data from Cohen and Schwartz (1983), Cohen et al. (1984), and Davidson and Jaffe (1984); the theoretical model corresponding to the dashed curve assumes $a=0.35 \mathrm{~km} / \mathrm{s}$, $M=0.5 M_{\odot}$ and $\Omega=5 \times 10^{-13} \mathrm{rad} / \mathrm{s}$; that of the dashed-dotted curve, to $a=0.325 \mathrm{~km} / \mathrm{s}$, $M=0.675 M_{\odot}$, and $\Omega=1 \times 10^{-13} \mathrm{rad} / \mathrm{s}$; and that of the solid curve to $a=0.35 \mathrm{~km} / \mathrm{s}$, $M=1.0 M_{\odot}$, and $\Omega=1 \times 10^{-13} \mathrm{rad} / \mathrm{s}$ with $\left(\eta_{*}, \eta_{D}\right)=(1.0,0.5)$ instead of $(0.5,1.0)$. 

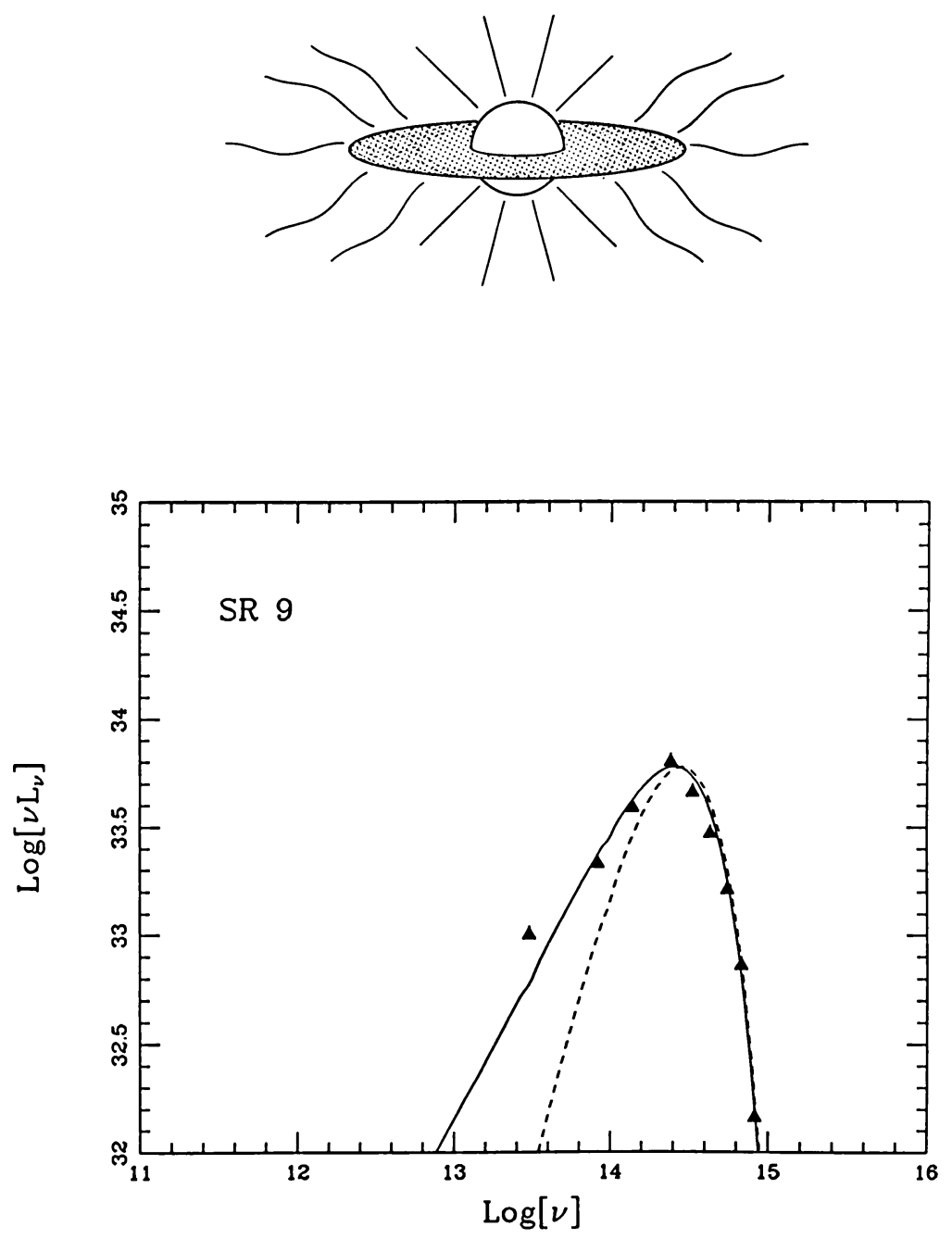

Figure 3. Theoretical and observed spectra of $\mathrm{T}$ Tauri star with infrared excess - SR 9 (Ophiuchus): data from Chini (1981) and Lada and Wilking (1984); theoretical model assumes $L=3.0 L_{\odot}, A_{V}=1.0 \mathrm{mag}$. and $T_{*}=4000 \mathrm{~K}$. A reddened black body (dashed line) is shown for comparison. 
the existence of narrow twin exhaust jets in the youngest outflow objects becomes a natural part of protostellar evolution.

The issue of whether the observed optical jets represent this phenomenon is more contentious. At least two alternatives can be envisaged. The first is that an observed YSO outflow starts from within a few stellar radii of the stellar surface already highly collimated, and it later opens up (perhaps because of insufficient radiative cooling as the shocked jets propagate down a declining density gradient in the ambient molecular cloud core) to sweep out the fatter lobes of $\mathrm{CO}$ emission observed by millimeter-wave radio astronomers (e.g., Fig. 4 of Shu, Lizano, and Adams 1987). The second is that a YSO outflow starts out from the stellar surface more-or-less as an isotropic stellar wind, but it later becomes focused through interactions with an anisotropic and inhomogeneous ambient medium (e.g., Fig. 2 of Konigl 1982). Klein, Sandford, and Shu (1986) are in the process of evaluating the viability of these alternative scenarios.

\section{T TAURI STARS WITH CIRCUMSTELLAR DISKS}

As time proceeds, we expect more and more of the rotating inflowing matter to fall preferentially on the disk rather than on the star. In any reasonable picture of stellar outflows, we expect the opening angle of the wind to widen with time, eventually sweeping outwards over all $4 \pi$ steradians. This marks the beginning of the fourth evolutionary phase - an optically visible T Tauri star, newly born on a convective (Hayashi) pre-main-sequence track, surrounded by a remnant nebular disk (Elsasser and Staude 1978, Cohen 1983, Beckwith et al. 1984, Grasdalen et al. 1984, Simon et al. 1985), and bubbling with surface activity (Herbig 1962; Kuhi 1964; Calvet, Basri, and Kuhi 1984). The slow rotation rates observed for $\mathrm{T}$ Tauri stars (Vogel and Kuhi 1981), which were quite surprising when first discovered, may now be naturally attributed to the large magnetic braking associated with an earlier period of intense mass loss.

This picture provides a natural explanation for the infrared excesses commonly observed in the spectra of T Tauri stars. Even if the circumstellar disk surrounding a newly formed star has no intrinsic luminosity, Adams and Shu (1986) showed that an optically thick (but spatially thin) disk will intercept and reradiate $25 \%$ of the stellar luminosity. The resulting star/disk spectra are in close agreement with observed T Tauri stars with infrared excesses (see Figure 3). In these sources, the equilibrium temperature distribution in the disk is given approximately by $T \propto r^{-3 / 4}$, similar to the classical result for a Keplerian accretion disk (LyndenBell and Pringle 1974). The corresponding spectral energy distribution will assume a power-law form at long wavelengths given by $\nu L_{\nu} \propto \nu^{4 / 3}$.

However, there is another class of $\mathrm{T}$ Tauri stars (which includes $\mathrm{T}$ Tauri itself) with spectral energy distriubtions that are more nearly flat at long wavelenths, i.e., $\nu L_{\nu} \sim$ constant. These sources can be understood if the disk has an intrinsic luminosity comparable to the stellar luminosity and the disk temperature distribution 
falls off less steeply than the $T \propto r^{-3 / 4}$ law appropriate for a Keplerian accretion disk (see ALS). Since a flatter temperature distribution implies a flatter rotation curve in a viscously evolving axisymmetric system, these sources probably have extended mass distributions, i.e. disks whose masses are a non-negligible fraction of the total. The case for massive disks can be given a more robust argument. First we note that the objects in question must often have intrinsic disk luminosities of several $L_{\odot}$ in order to explain the observed infrared excesses. Accretion onto a star (either the central source or a companion embedded in the disk) is the most likely energy source and hence must occur at a rate of $10^{-5}$ to $10^{-6} M_{\odot} / y$. Since many $\mathrm{T}$ Tauri stars have such infrared excesses, and their lifetimes are on the order to $10^{5}$ to $10^{6} \mathrm{y}$, the reservoirs of material for accretion in the disks must often be $0.1-1.0 M_{\odot}$ (cf. Sargent and Beckwith 1986).

It is interesting to speculate on the modes of companion formation in the two extreme cases of $\mathrm{T}$ Tauri disks. If a nebular disk turns out to have enough mass to make another star, it may be an excellent candidate for forming a relatively close binary system (with a period shorter than $\sim 10^{2}-10^{3}$ y; see Abt 1983). A passive or a nearly passive disk will contain a smaller amount of material, and it may be a likely candidate for forming a planetary system.

\section{REFERENCES}

Abt, H. 1983, Ann. Rev. Astr. Ap., $21,343$.

Adams, F. C., and Shu, F. H. 1985, Ap. J., 296, 655.

Adams, F. C., and Shu, F. H. 1986, Ap. J., in press (AS).

Adams, F. C., Lada, C. J. and Shu, F. H. 1987, Ap. J., in press (ALS).

Appenzeller, I., and Tscharnuter, W. 1974, Astr. Ap., s0, 423.

Bally, J., and Lada, C. J. 1983, Ap. J., 265, 824.

Beckwith, S., Zuckerman, B., Skrutskie, M. F., and Dyck, H. M. 1984, Ap. J., 287, 793.

Bertout, C., and Yorke, H. W. 1978, in Protostars and Planets, ed. T. Gehrels (Tucson: University of Arizona Press), p. 648.

Bodenheimer, P., and Sweigart, A. 1968, Ap. J., 152, 515.

Bodenheimer, P. 1980, in IAU Symposium No. 93, Fundamental Problems in the Theory of Stellar Evolution, ed. D. Sugimoto, D. Q. Lamb, and D. N. Schramm (Dordrecht: Reidel), p. 5.

Burton, W. B. 1976, Ann. Rev. Astr. Ap., 14, 275.

Calvet, N., Basri, G., and Kuhi, L. V. 1984, Ap. J., 277, 725.

Cassen, P., Shu, F. H., and Terebey, S. 1985, in Protostars and Planets II, ed. D. C. Black and M. S. Matthews (Tucson: University of Arizona Press), p. 448. 
Chandrasekhar, S. 1939, An Introduction to Stellar Structure (The University of Chicago Press).

Chini, R. 1981, Astr. Ap., 99, 346.

Cohen, M. 1983, Ap. J. (Letters), 270, L69.

Cohen, M. 1984, Physics Reports, 116, no. 4, 173.

Cohen, M., and Kuhi, L. V. 1979, Ap. J. Suppl., 41, 743.

Cohen, M., Harvey, P. M., Schwartz, R. D., and Wilking, B. A. 1984, Ap. J., 278, 671.

Cohen, M., and Schwartz, R. D. 1983, Ap. J., 265, 877.

Davidson and Jaffe, D. T. 1984, Ap. J. (Letters), 277, L13.

Elmegreen, B.G. 1979, Ap. J., 232, 729.

Elmegreen, B.G. 1986, preprint.

Elsasser, H., and Staude, H. J. 1978, Astr. Ap., 70, L3.

Goodman, A. 1986, private communication.

Grasdalen, G. L., Strom, S. E., Strom, K. M., Capps, R. W., Thompson, D., and Castelaz, M. 1984, Ap. J. (Letters), L57.

Hayashi, C., Hoshi, R., and Sugimoto, D. 1962, Prog. Theor. Phys. Suppl. No. 22.

Herbig, G. 1962, Adv. Astr. Ap. 1, 47.

Klein, R. I., Sandford, M. T., and Shu, F. H. 1986, in preparation.

Klein, R. I., Whitaker, R. W., and Sandford, M. T. 1985, in Protostars and Planets II, ed. D. C. Black and M. S. Matthews (Tucson: University of Arizona Press), p. 340.

Konigl, A. 1982, Ap. J., 261, 115.

Kuhi, L. V. 1964, Ap. J., 140, 409.

Lada, C. J. 1985, Ann. Rev. Astr. Ap., 23, 267.

Lada, C. J., and Wilking, B. A. 1984, Ap. J., 287, 610.

Larson, R. B. 1969, M. N. R. A.S., 145, 271.

Larson, R. B. 1969, M. N. R. A. S., 145, 297.

Lizano, S., and Shu, F. H. 1986, in preparation.

Martin-Pintado, J., Wilson, T. L., Gardner, F. F., and Henkel, C. 1983, Astr. Ap., 117, 145.

Mercer-Smith, J. A., Cameron, A. G. W., and Epstein, R. I. 1984, Ap. J., 287, 445. 
Mundt, R., and Fried, J. W. 1983, Ap. J. (Letters), 274, L83.

Mundt, R., Walter, F. M., Feigelson, E. D., Finkenzeller, U., Herbig, G. H., and Odell, A. P. 1983, Ap. J., 269, 229.

Myers, P. C., and Benson, P. J. 1983, Ap. J., 266, 309.

Myers, P. C. 1987, in Star Forming Regions, ed. M. Peimbert and J. Jugaku (Dordrecht: Reidel).

Nakano, T., and Umebayashi, T. 1980, Pub. Astr. Soc. Japan, 32, 613.

Nakano, T. 1981, Prog. Theor. Phys. Suppl. No. 70, 54.

Nelson, L. A., Rappaport, S. A., and Joss, P. C. 1986, Ap. J., in press.

Parker, E. N. 1979, Cosmical Magnetic Fields (Oxford University Press).

Sargent, A., and Beckwith, S. 1986, in preparation.

Scoville, N. Z. 1986, in Star Formation in Galaxies, ed. G. Neugebauer and N. Z. Scoville, in press.

Shu, F. H. 1977, Ap. J., 214, 488.

Shu, F. H. 1985, in The Milky Way, ed. H. van Woerden, W. B. Burton, R. J. Allen (Dordrecht: Reidel), p. 561.

Shu, F. H. 1986, in Star Formation in Galaxies, ed. G. Neugebauer and N. Z. Scoville, in press.

Shu, F. H., and Terebey, S. 1984, in Cool Stars, Stellar Systems, and the Sun, ed. S. Baliunas and L. Hartmann (Berlin: Springer-Verlag), p. 78.

Shu, F. H., Lizano, S., and Adams, F. C. 198飞, in Star Forming Regions, ed. M. Peimbert and J. Jugaku (Dordrecht: Reidel).

Solomon, P. M. and Sanders, D. B., in Protostars and Planets II, ed. D. C. Black and M. S. Matthews (Tucson: University of Arizona Press), p. 59.

Stahler, S. W. 1983, Ap. J., 274, 822.

Stahler, S. W., Shu, F. H., and Taam, R. E. 1980, Ap. J., 241, 637 (SST).

Stahler, S. W., Shu, F. H., and Taam, R. E. 1980, Ap. J., 242, 226.

Stahler, S. W., Shu, F. H., and Taam, R. E. 1981, Ap. J., 248, 727.

Terebey, S., Shu, F. H., and Cassen, P. 1984, Ap. J., 286, 529.

Vogel, S., and Kuhi, L. 1981, Ap. J., 245, 960.

Wadiak, E. J., Wilson, T. L., Rood, R. T., and Johnston, K. J. 1985, Ap. J. (Letters), 295, L43. 
Walter, F. W. 1986, Pub. Astr. Soc. Pac., in preparation.

Walmsley, M. 1988, this volume.

Whitworth, A., and Summers, D. 1985, M. N. R. A. S., 214, 1.

Wilking, B. A., and Lada, C. J. 1983, Ap. J., 274, 698.

Winkler, K. H., and Newman, M. J. 1980, Ap. J., 236, 201.

Yorke, H. W., and Krugel, E. 1977, Astr. Ap., 54, 183.

Yorke, H. W., and Shustov, B. M. 1981, Astr. Ap., 98, 125.

Young, E. T., Lada, C. J., and Wilking, B. A. 1986, Ap. J. (Letters), in press.

Zeng, Q., Batrla, W., and Wilson, T. L. 1984, Astr. Ap., 141, 127.

Zuckerman, B., and Palmer, P. 1974, Ann. Rev. Astr. Ap., 12, 279. 